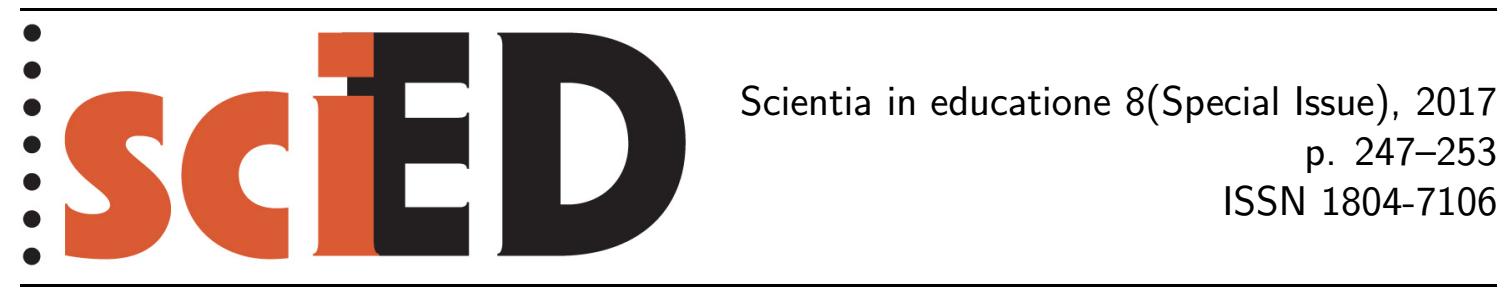

\title{
Just How Deterring Are Formulas?
}

\section{Alexander Strahl, Rainer Müller, Susan Hagendorf, Julian Grobe}

\begin{abstract}
Formulas are an effective means for communication in physics. Most teachers would agree, however, that novices tend to be deterred by formulas. Up to now, this common belief has never been substantiated by quantitative research. Here we report on an attempt to identify and quantify the variables that govern the appraisal of physical formulas. In an empirical study, 684 secondary school and university students were asked to indicate for 38 formulas to which extent they perceive the formula as deterring. The result is surprisingly simple. We are able to model the responses with only a single variable: the length of the formula. An explicit model equation (saturating exponential) to fit the data can be given.
\end{abstract}

Key words: formula, unit, deterring, length of formula questionnaire study. 


\section{INTRODUCTION}

Previous studies on physical formulas concentrated mainly on their role in text comprehension and problem solving. Dee-Lucas and Larkin (1988) found that undergraduate physics students judged physical texts containing formulas as more important than their verbal counterparts. The same authors found a slight advantage in text comprehension when the formulas in a physics text were replaced by verbal equivalents (Dee-Lucas \& Larkin, 1991). This result was called into question by Müller and Heise (Müller \& Heise, 2006), who found a significant advantage in text comprehension for secondary school students reading the version with formulas. Remarkably, most of the students interviewed by Müller and Heise expressed a positive attitude towards physical formulas, just as in Strahl et al. (2009, 2010).

The role of formulas in problem solving has been explored in the context of expert/novice research. There is evidence that experts and novices solve physics problems differently. According to Larkin et al. (1980) and Larkin (1983) novices tend to use formulas in the early stages of problem solving, while experts develop a qualitative representation before using equations.

Perhaps the most famous remark on the subject of the present note has been made by Stephen Hawking. In the preface of his popular book "A brief history of time" (Hawking, 1988), he writes: "Someone told me that each equation I included in the book would halve the sales. I therefore resolved not to have any equations at all. In the end, however, I did put in one equation, Einstein's famous equation $E=m c^{2}$. I hope this will not scare off half of my potential readers." Presumably, there are two reasons why Hawking did not fear that this particular formula would deter his readers too much: (a) he could assume the readers are familiar with it and (b) it is not too complicated.

\section{SETTing OF THE STUDY}

In our empirical study, we asked students to indicate for 38 formulas to which extent they perceived the formula as deterring. The formulas were taken from different fields of physics, with varying length and complexity. Some examples are:

$$
\begin{aligned}
s & =\frac{a \cdot t^{2}}{2} \\
f & =\frac{1}{2 \pi \sqrt{L \cdot C}} \\
W & =\int F \cdot d r \\
u_{\nu}(\nu, T) & =\frac{8 \pi v^{2}}{c^{3}} \frac{h \nu}{\mathrm{e}^{\frac{h \nu}{k T}}-1} \\
\Delta K_{\text {kin }} & =\frac{1}{2}\left(m_{1} \cdot v_{1}^{2}+m_{2} \cdot v_{2}^{2}\right)-\frac{1}{2} u^{2}\left(m_{1}+m_{2}\right)
\end{aligned}
$$

We interrogated three different groups of students:

Group 1: A random sample of 288 secondary school students (grade 10 to 12),

Group 2: 258 first-year university students not majoring in physics,

Group 3: 24 physic education students for middle school,

Group 3: 114 first-year physics and electrical engineering majors.

The participants had to complete a questionnaire in which they rated each of the 38 formulas on a scale from 1 (not at all deterring) to 5 (very deterring). For 
quantitative modeling it is more convenient to use a scale that varies from 0 to 1 . The data were thus rescaled by a linear transformation. In total, we obtained 25992 individual ratings from the 684 participants. The group averages of these ratings define a "degree of deterrence" for the 38 formulas.

\section{ThOught}

At first sight, it seems quite hopeless to predict how the students would assess the formulas. There is an abundance of factors that may affect the rating:

1. the familiarity of the students with the subject area to which the formula belongs,

2. the level of physics expertise of the students,

3. the familiarity with the formula itself or with the variables contained in it,

4. the appearance of unusual symbols (Greek letters, square roots or integral signs),

5. the length of the formula,

6. the structure of the formula (appearance of brackets, fraction bars).

Factors 1 and 3 can be controlled by inspection of the physics curricula of the different groups. The level of expertise can roughly be assessed by the group membership and the last physics grade. On the contrary, it is not entirely obvious how to define the notion "formula length". We chose the simplest definition we could think of: counting the number of symbols appearing in the formula. Any symbol, be it a letter, a number, a fraction bar, or a plus sign, contributes equally to the length. Functions like sin, cos, or exp and named indices (like the index "kin" in (5)) are counted once. For the formulas (1)-(5) shown above, we obtain a length of 8, 10, 7 , 26 , and 35 , respectively.

\section{RESULT — FORMULA}

Surprisingly the responses of each group could be modelled with a single variable: the formula length defined above. The remaining factors seem to have a much smaller influence on the degree of deterrence. Figure 1 shows the data of group 2 plotted as a function of formula length. A marked nonlinear relationship is clearly discernible. The graph suggests that the length alone appears to be a good indicator of how deterring a formula is perceived.

The data can be interpreted as follows: Short formulas are perceived as less deterring than longer ones. The relation is not linear, however. Increasing the formula length by 5 symbols has a stronger effect for a formula of length 5 than for one with length 20. The deterring effect saturates.

Saturation phenomena are known from physics and many other branches of science. Perhaps the simplest example is the charging of a capacitor. Quantitatively, these phenomena are typically described by a saturating exponential of the form $1-\mathrm{e}^{-x}$. An analogous model appeared to be promising for the introduced correlation.

Using a nonlinear least-square method, the data was fitted to the model equation

$$
y=1-\mathrm{e}^{-\frac{x-3}{A}} .
$$




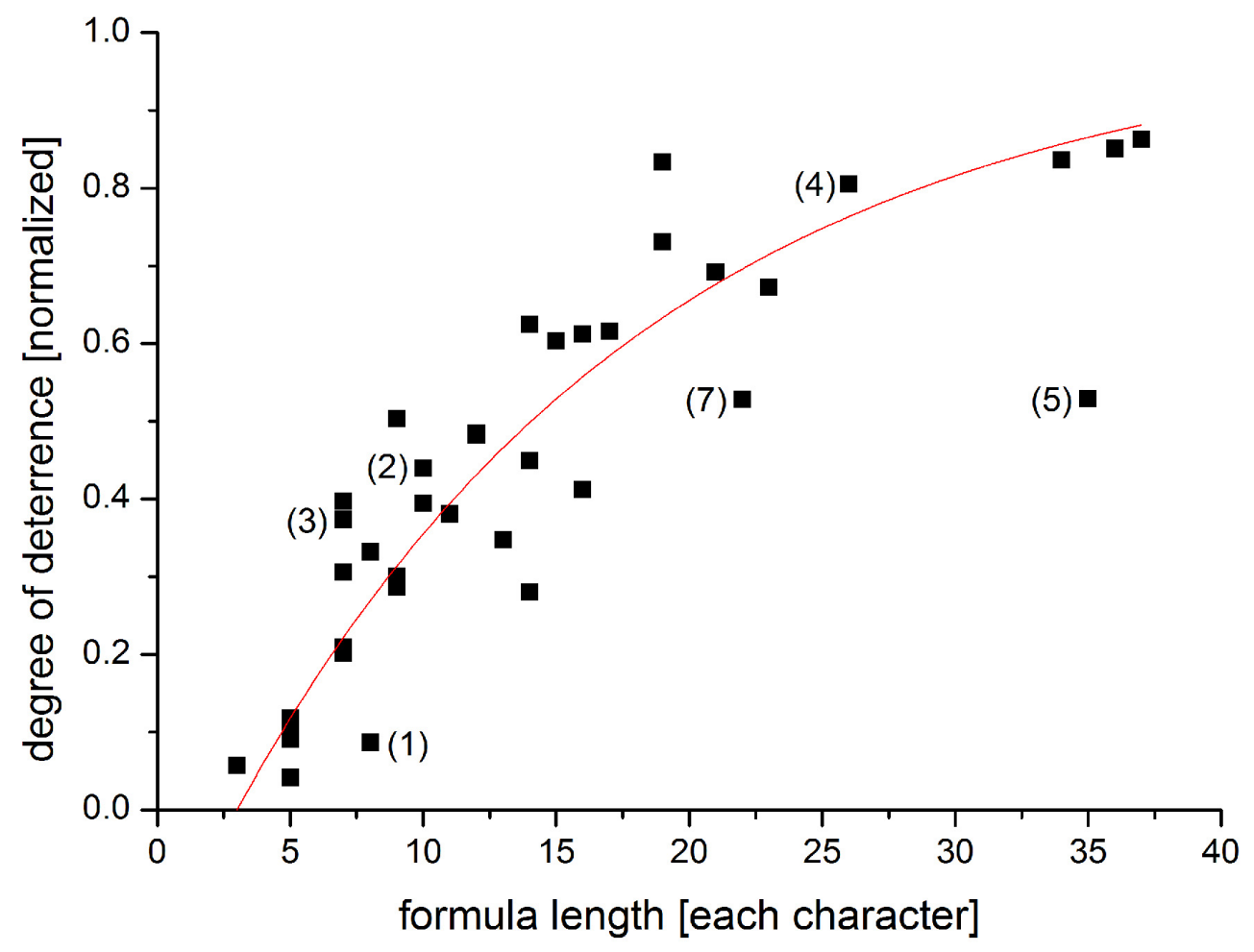

Figure 1: Degree of deterrence vs. formula length for the 38 formulas rated by the students of group 2. The degree of deterrence is defined as the average student rating of the formula within the group, rescaled to the interval $(0,1)$. The solid line is the best fit curve for the model (6). The labels (1)-(5) and (7) mark the formulas shown in the text

The fit function intersects the abscissa at $x=3$, reflecting the fact that this is the smallest conceivable length of a formula (e. g. $a=b$ ). The parameter $A$ determines the slope of the curve and can be interpreted as a saturation length.

The fitting was done for each group separately. The data point marked with (5) was classified as an outlier and excluded from the analysis. We will return to the interpretation of this point below. Without the outlier, the hypothesis that the fit follows a Gaussian distribution is consistent with the data.

The solid line in Figure 1 shows the curve that best fits the data for group 2 . Table 1 lists the corresponding value of $A$ together with common measures for the goodness of the fit. It is remarkable, how well the students responses can be modelled with a single free parameter. The standard error of estimate, for example, is about 0.1. It can be interpreted as the average distance of the data points from the fitting curve.

Table 1: Fit parameters and goodness-of-fit measures for the four groups. Note that, unlike for linear models, it is not possible to interpret $R^{2}$ as the percentage of the variance explained by the model

\begin{tabular}{l|c|c|c|c|c|c|c|c}
\cline { 2 - 11 } & \multicolumn{4}{c|}{ formula } & \multicolumn{5}{c}{ unit } \\
\cline { 2 - 11 } & $A$ & $s$ & $R^{2}$ & $n$ & $B$ & $S$ & $R^{2}$ & $n$ \\
\hline Student (school) & 10.75 & 0.70 & 0.76 & 288 & 10.69 & 1.82 & 0.26 & 143 \\
\hline subsidiary subject (university) & 15.95 & 1.03 & 0.78 & 258 & 14.96 & 2.97 & 0.46 & 304 \\
\hline teaching physics (university) & 16.75 & 0.99 & 0.86 & 24 & & & & \\
\hline physics student (university) & 30.42 & 2.26 & 0.72 & 114 & & & & \\
\hline
\end{tabular}




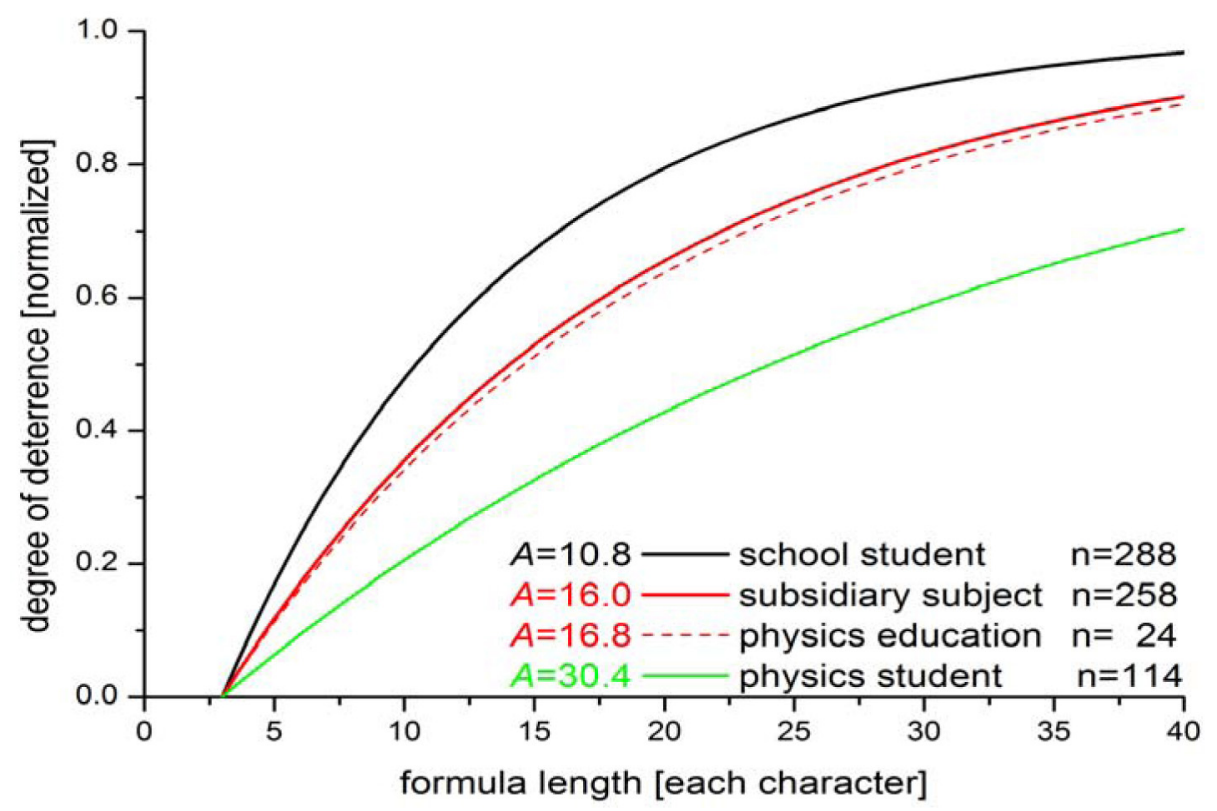

Figure 2: Best fitting curves for the four groups. The characteristic length for each curve can be found in Table 1

Let us finally comment on the rating of Eq. (5). The perceived degree of deterrence is much lower than expected (data point (5) in Figure 1). We believe to see an instance of chunking here. In psychology, chunking designates the ability to group several objects into a larger meaningful units (Chase \& Simon, 1973). Eq. (5) consists of several similar terms that can be interpreted as kinetic energies. Because of chunking, the formula may be perceived to consist of "less elements", leading to an apparent reduction of complexity. To a lesser extent, such an effect can also be seen for the formula marked (7)

$$
E=\frac{n^{2}}{8 m a^{2}}\left(n_{x}^{2}+n_{y}^{2}+n_{z}^{2}\right)
$$

where repeating elements may lead to a lower rating. These effects, together with a more detailed analysis of the influence of the other factors mentioned above, are subject to ongoing research.

\section{RESULT - UNIT}

A follow-up study has been carried out with physical units (like $\mathrm{N} \cdot \mathrm{m}$ or $\mathrm{V} \cdot \mathrm{s} /(\mathrm{A} \cdot \mathrm{m})$ ). Here we could find similar correlations between the length of a unit and the degree of deterrence (see Figure 3 and Table 2).

Table 2: Different degree of deterrence for the same unit (from 0 to 1 )

\begin{tabular}{c|c|c}
\hline unit & number of symbols & $\begin{array}{c}\text { average degree } \\
\text { of deterrence }\end{array}$ \\
\hline $\mathrm{T}=\frac{\mathrm{V} \cdot \mathrm{s}}{\mathrm{m}^{2}}$ & 8 & 0.31 \\
\hline $\mathrm{T}=(\mathrm{V} \cdot \mathrm{s}) / \mathrm{m}^{2}$ & 10 & 0.38 \\
\hline $\mathrm{T}=\mathrm{V} \cdot \mathrm{s} \cdot \mathrm{m}^{-2}$ & 9 & 0.42 \\
\hline
\end{tabular}




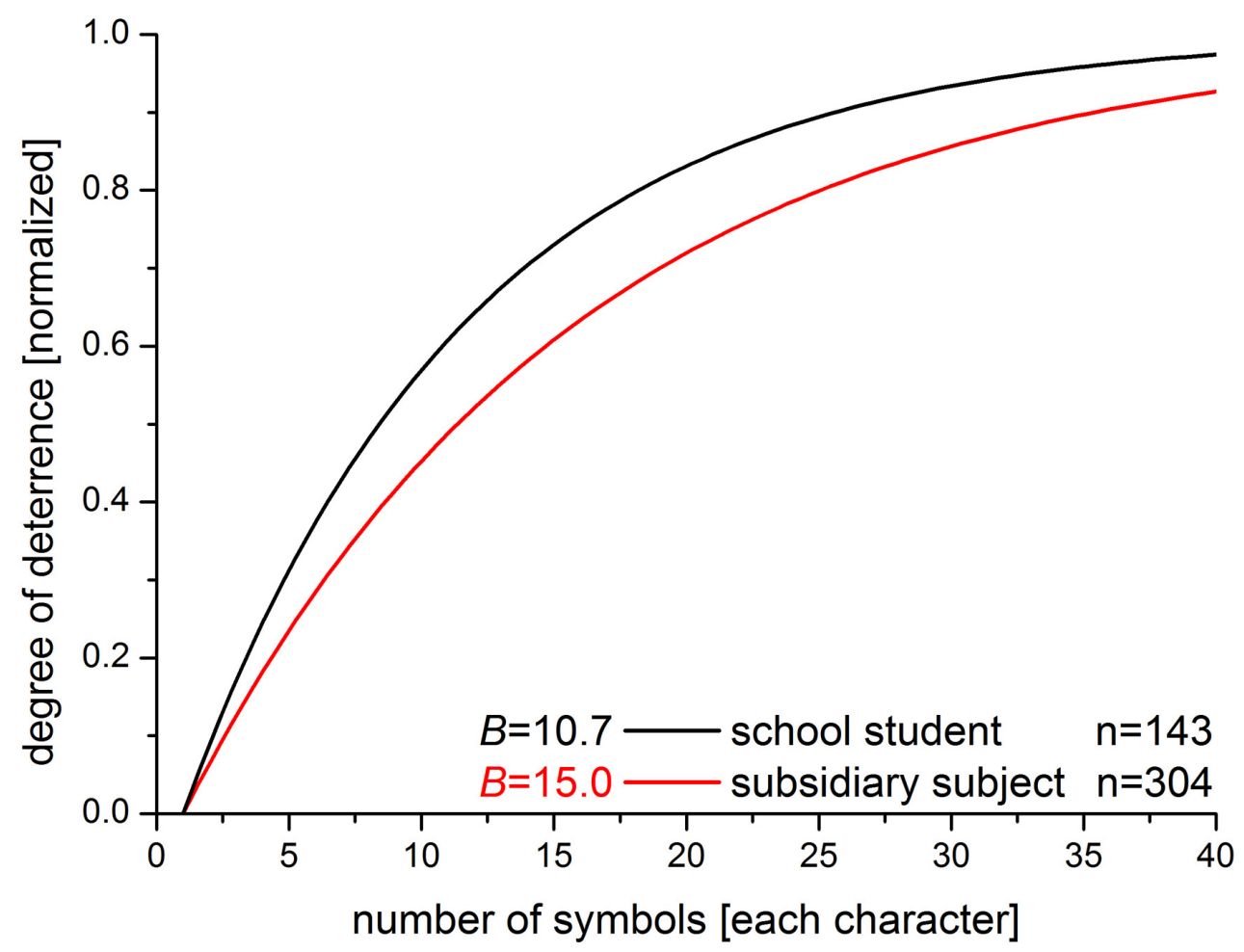

Figure 3: Fitting curve for the deterrence of units

Figure 3 shows the best-fitting curves for a group of school students ( $n=143$ ) and a group of university students with physics as subsidiary subject $(n=304)$ who assessed the degree of deterrence of 22 units. As in the study on formulas, the length of a unit is determined by the number of its symbols.

The degree of deterrence of units demands a slight modification of the model equation that fitted the data for the length of formulas. The data for units could be fitted to the equation

$$
y=1-\mathrm{e}^{-\frac{x-1}{B}} .
$$

The fit parameter $B$ determines the slope of the curves. As shown in Table 1, it differs for school and university students.

In the study on units, we further obtained some interesting results with regard to different representations of fractions. Table 2 shows three different representations of the same fraction. The first of them is preferred by the students.

Table 2. Different degree of deterrence for the same unit (from 0 to 1 )

Further research carried out along these lines (Strahl, Grobe \& Müller, 2010) shows that students prefer certain representations of formulas (like a horizontal bar in fractions or writing out the indices within a formula).

\section{REFERENCES}

Chase, W. G. \& Simon, H. A. (1973). The mind's eye in chess. In Chase, W. G. (Ed.), Visual information processing. New York: Academic Press.

Dee-Lucas, D. \& Larkin, J. H. (1988). Novice rules for assessing importance in scientific texts. Journal of Memory and Language, 27(3), 288-308. 
Dee-Lucas, D. \& Larkin, J.H. (1991). Equations in Scientific Proofs: Effects on comprehension. American Educational Research Journal, 28(3), 661-682.

Hawking, S. A. (1988). Brief history of time. New York: Bantam Dell.

Larkin, J. H., McDermott, J., Simon, D. P. \& Simon, H. A. (1980). Models of competence in solving physics problems. Cognitive Science, 4(4), 317-345.

Larkin, J. H. (1983). The role of problem representations in physics. In Gentner, D. \& Stevens, A. L. (Eds.), Mental models (75-98). Hillsdale: Erlbaum.

Müller, R. \& Heise, E. (2006). Formeln in physikalischen Texten: Einstellung und Textverständnis von Schülerinnen und Schülern. PhyDid, 2(5), 62-70.

Strahl, A., Grobe, J. \& Müller, R. (2010). Was schreckt bei Formeln ab? Untersuchung zur Darstellung von Formeln. In PhyDid B.

Strahl, A., Mohr, M., Schleusner, U. \& Müller, R. (2009). Beurteilung von Formeln durch Schüler - eine Fragebogen-Untersuchung. In D. Höttecke (Ed.), Chemie- und Physikdidaktik für die Lehramtsausbildung. Gesellschaft für Didaktik der Chemie und Physik. Jahrestagung in Schwäbisch Gmünd 2008. Münster: Lit-Verlag.

Strahl, A., Mohr, M., Schleusner, U., Krecker, M. \& Müller, R. (2010). Akzeptanz von Formeln - Vergleich zweier Erhebungen. In PhyDid B.

Alexander STRAHL

RAINER MÜLLER

SusAN HAGENDORF

JuLian Grobe

TU Braunschweig, Germany 